\title{
Fault Detection in the Activated Sludge Process using the Kohonen Self-Organising Map
}

\author{
Rabee Rustum ${ }^{1} \&$ Shaun Forrest ${ }^{2}$ \\ ${ }^{1}$ School of Energy, Geoscience, Infrastructure and Society, Heriot-Watt University, UK, Dubai Campus, Dubai \\ International Academic City, P O Box 294345, Dubai, UAE. \\ r.rustum@hw.ac.uk \\ ${ }^{2} \mathrm{PhD}$ Student, Carleton University, Ottawa, Canada \\ shaunforrest@gmail.com
}

\begin{abstract}
This paper addresses the detection of faulty situations that develop in activated sludge wastewater treatment plants (ASWWTP). The Kohonen Self-Organising map (KSOM) has been utilised to detect and track changes in different parameters for real data collected from the Seafield wastewater treatment plant, Edinburgh, $U K$. The results demonstrate that this method is simple, computationally efficient and provides useful information for the process engineer who is faced with improving the performance of the WWTP.
\end{abstract}

Keywords: Wastewater treatment, Fault detection, activated sludge, Kohonen Self Organising Map

\section{Introduction}

Increased regulations which protect the environment and water bodies, have led to a growing demand for the improved performance of wastewater treatment plants. This reality has encouraged environmental engineers to use innovative techniques to improve plant operation and control through designing systems that describe the dynamic behaviour of the treatment plant through both qualitative and quantitative methods. Such systems help the process engineer to convert unsatisfactory dynamic behaviour to satisfactory behaviour, thus reducing operational costs and meeting the requirements of regulatory agencies while minimising any adverse effects on the environment [1].

However, controlling the activated sludge system is very complex due to factors such as: a high temporal variability in flow and concentrations of the influent; complex and poorly known relations between the variables; the variation over time of living microorganisms in quality and the number of species; the limited detailed knowledge of biological treatment mechanisms; scarce and often unreliable use of online analysis; delays in obtaining the analytical results of some parameters; difficulty of using human experts as the knowledge and experience gained from operational complications of one wastewater treatment plant, cannot be easily applied to another. These characteristics make it difficult to control the activated sludge process. Hence, it has been necessary to develop methods to improve the robustness of monitoring and fault detection [2][3].

Fault detection is the process by which a fault is identified, whereas diagnosis involves identifying the type of fault and its cause [4]. However, due to the highly complex activated sludge process, process disturbances are usually difficult to detect. For example, under normal operating conditions, the principal aim of the plant is to maintain the effluent concentration below a certain reference value. However, there are many situations that can inhibit the plant from achieving this aim, including (but not limited to); changes in the mixed liquor suspended solids, the presence of heavy metals and/or filamentous, bulking phenomena, problems in the piping system and a high concentration of dissolved oxygen. These changes must be detected and diagnosed quickly to prevent faults in the WWTP. Moreover, the measurement system itself can be defective due to the hostile and highly 
dynamic conditions at sensor locations or laboratory equipment. The measurement is declared inaccurate when it produces a signal that is outside the permitted range of values of the parameter that is being monitored.

In modern control theory, fault detection and diagnosis are predominantly based on redundancy, which employs a model of the monitored system. These methods utilise dynamic models to detect the fault [5]. According to these methods, faults are presumed to appear as changes within different components of the plant. These changes can successfully be determined by incorporating estimation techniques. However, the challenge with these approaches is that they require robust models to represent the plant under various conditions including fault situations, which are not easy in practice, as the fault situations might be very rare during training and validation stages of model construction. Moreover, most of these models have been proposed to estimate the dynamic behaviour of both the biological reactor and the secondary settler, whereas very few models incorporate the interconnection between both of these units [1]. In addition, these models were developed using data obtained under controlled laboratory conditions and are therefore, more suitable for the design of treatment plants rather than operational control, with very few of the models validated with real field data [6]. In recent years, numerous researchers have undertaken fault detection studies in wastewater treatment [7][8]. However, very few applications used the Kohonen self-organising map (KSOM) to detect faults in activated sludge wastewater treatment plants [3], [9]. The KSOM has been used for a wide variety of applications, however, the most important application of the KSOM has been in the visualisation of high-dimensional systems and process data and the discovery of categories and abstraction from raw data[3][10]. A variety of neural networks have been used in WWTP research, with significant attention directed towards supervised neural networks [5]. However, there is less focus in applying KSOM in the field of wastewater treatment [11]. This work describes the use of KSOM, unsupervised artificial neural networks, to detect faulty situations in activated sludge processes.

\section{Fault Detection using the Kohonen Self-Organizing Map (KSOM)}

The KSOM is one of the most widely employed artificial neural network algorithms (Kohonen et al. 1996). It is usually presented as a dimensional grid or map whose units (nodes) become tuned to different input data patterns. Its algorithms are based on unsupervised competitive learning, which means that training is entirely data driven and the neurons or nodes on the map compete with each other [3].

The principal goal of the KSOM is to transform an incoming signal pattern of arbitrary dimensions into a two-dimensional discrete map. It involves clustering the input patterns in such a way that similar patterns are represented by the same output neuron, or by one of its neighbours. In this way, the KSOM can be viewed as a tool for reducing the amount of data by clustering, thus, converting complex, nonlinear statistical relationships between high dimensional data, into simple relationships on low dimensional display (Kohonen et al., 1996). This mapping roughly preserves the most important topological and metric relationship of the original data elements, implying that minimal information is lost during the mapping.

The KSOM consists of two layers: the multi-dimensional input layer and the competitive or output layer, both of which are fully interconnected. The output layer consists of M neurons arranged in a two-dimensional grid of nodes. Each node or neuron $\mathrm{i}(\mathrm{i}=1,2, \ldots, \mathrm{M}$, where $\mathrm{M}$ is the total number of neurons, i.e. the size of the map) is represented by an $n$-dimensional weight or reference vector $m_{i}=\left[m_{i 1}, \ldots \ldots, m_{i n}\right]$, where $n$ is the dimension of the input vectors. Therefore, the weight vectors of the KSOM form a codebook and their neurons are connected to adjacent neurons by a neighbourhood function which dictates the structure of the map. In other words, the M nodes can be ordered so that similar neurons are located together, and dissimilar neurons are remotely located on the map. This technique is highly visual, and the topology of the map may be structured as a rectangular or hexagonal grid. Additional details on map training and validation can be found in [1].

Fault detection using KSOM is based on monitoring the residual generation, produced by comparing the input vectors with their corresponding best map unit (BMUs). This residual is called 'quantisation error'. If the residual exceeds specified thresholds, then a fault is highly likely, and the process may be defective. This 
conclusion is based on the assumption that a large quantisation error corresponds to the operation point belonging to measurement space not covered by the training data that describe normal operational conditions. Therefore, the situation is new, and something is possibly going wrong.

\section{Application}

A data record from a large wastewater treatment plant in Edinburgh, UK has been utilised in this study. Faulty situations at the plant are examined in order to validate the method and to study their reliability when applied to a real process.

\subsection{Experimental Data}

Daily records from the operation of the Seafield wastewater treatment plant in Edinburgh, UK were collected during a period of approximately three years with a total of $\mathrm{N}=1066$ data samples (or records), with each data sample comprised of 14 variables. The Seafield treatment plant adopts an activated sludge secondary treatment system. Initially, the raw data is screened or filtered to remove corrupt data such as missing values, noise and outliers [3].

\subsection{KSOM Analyses}

The SOM toolbox for Matlab 5 was used in the study. The computation for training and searching for the Best Map Units (BMUs) was done using batch training algorithms. Detailed guidelines on how to actually compute the map size and search for the BMU is described in the SOM Toolbox for Matlab 5 [12]. As known from the activated sludge process, normal situations dominate the available process data. However, some faulty observations are found and filtered out from the training dataset to accurately analyse normal operation. In order to detect faulty situations, we have trained the KSOM using the records of variables describing normal plant operation. Vectors representing abnormal behaviour of the processes are removed from the training data set. Therefore, a map describing normal operating conditions has been created. In the second phase, a set of faulty situations was carried out from the real data in order to test the approach.

\section{Results and Discussion}

This paper presents a method for detecting faults in wastewater treatment plants based on an activated sludge system. It combines a simple algorithm based on Kohonen Self Organising Map Technique. The characteristics of the trained map is highlighted in Table 1.

TABLE I: Characteristics of the trained KSOM

\begin{tabular}{|l|l|}
\hline Normalisation method & "var": $x^{\prime}=(x-\bar{x}) / \sigma_{x}$ \\
\hline Training Data & $1066 \times 14$ \\
\hline Codebook & $165 \times 14$ \\
\hline Neighbourhood function & Gaussian \\
\hline M size & $15 \times 11$ \\
\hline Lattice & "Hexa" \\
\hline Shape & Sheet \\
\hline Final quantisation error & 1.915 \\
\hline Final topographic error & 0.055 \\
\hline
\end{tabular}

In order to assess this method, an off-line simulation study was performed using 1066 data records. Different faulty situations in some parameters are presented and the KSOM is implemented to detect them. By measuring the distance between the new vector and its BMU using Euclidian distance, one can detect the abnormal situation. However, there is a need to examine this case carefully by some other means. For example, from the time series plots of the quantisation error for $\mathrm{BOD}_{5}$ (Fig 1), one can see some points rising above or below the upper and lower thresholds. This indicates large errors as the process behaviour is different from the normal data 
that was used for training the map. In another words, if the quantisation error is within the range, then we have normal operation conditions. Otherwise, we have an abnormal situation and the data needs to be investigated with greater detail. For example, the component planes for each of the 14 variables with the experimental data are displayed in Fig. 2. Each component plane can be thought as a "sliced" version of the KSOM, because it consists of values of a single vector variable in all map units. In other words, the component planes show the value of the variables in each map unit. These component planes help to illustrate visually the correlation between the various parameters or characteristics of the wastewater treatment plant.

The visualisation capability of the KSOM allows not only picturing the correlation of raw data in a two dimensional discreet map, but also allows monitoring the state of the process. Monitoring the process is based on the tracking of operation points in addition to the location of the point on the map indicating the process state. In another words, the state of the process can be monitored by drawing a pointer that displays the neuron corresponding to the latest measurement vector. Additionally, this method is advantageous to monitor how the state of the process has evolved during the last measurements, and therefore, a suitable length trajectory of the latest neurons, representing the latest operational states, can be drawn on the map. With this kind of transformation, it would be possible for the operator to visually follow the development of the system state. If the movement over one of the component planes moves sharply from two remote neurons, then a fault has occurred, and an alarm might be issued, or some of the map areas might be marked as alarmed states.

In case 2 for example, a failure of the process occurs on data number 60 on the 29/06/02. One can see that while the values are normal, the trajectory stays neutral and when the values begin to rise, it moves towards the alarming area. With this in mind, it would be possible for the operator of the wastewater treatment plant to visually follow the development of the system state, thus, the control of the system could be based on the state analysis.

\section{Conclusion}

In this paper, different faulty situations in some of the parameters are presented and the KSOM that has been trained with normal operation condition data is implemented to detect them. Normal operating samples were associated with their best map units (BMUs), then corresponding elements from the sample and the BMU are compared to find thresholds of normal operational conditions. Additionally, the BMU for faulty data are found and compared with their corresponding sample. To determine if the sample describes normal or faulty situations, we assigned upper and lower thresholds for the quantisation error for each element. If the quantisation error is inside threshold limits, then it describes normal operation conditions, otherwise, it describes a faulty situation. Once a problem has been detected, the logical step is to identify and diagnose the cause of the problematic event. If the fault is with a sensor or in the laboratory equipment, rather than the process itself, then we need to estimate the normal value from its BMU, otherwise a correction is needed to keep the plant in a desired situation. The method is simple and computationally efficient, and it provides useful information for the process engineer who is faced with improving the performance of WWTP. 


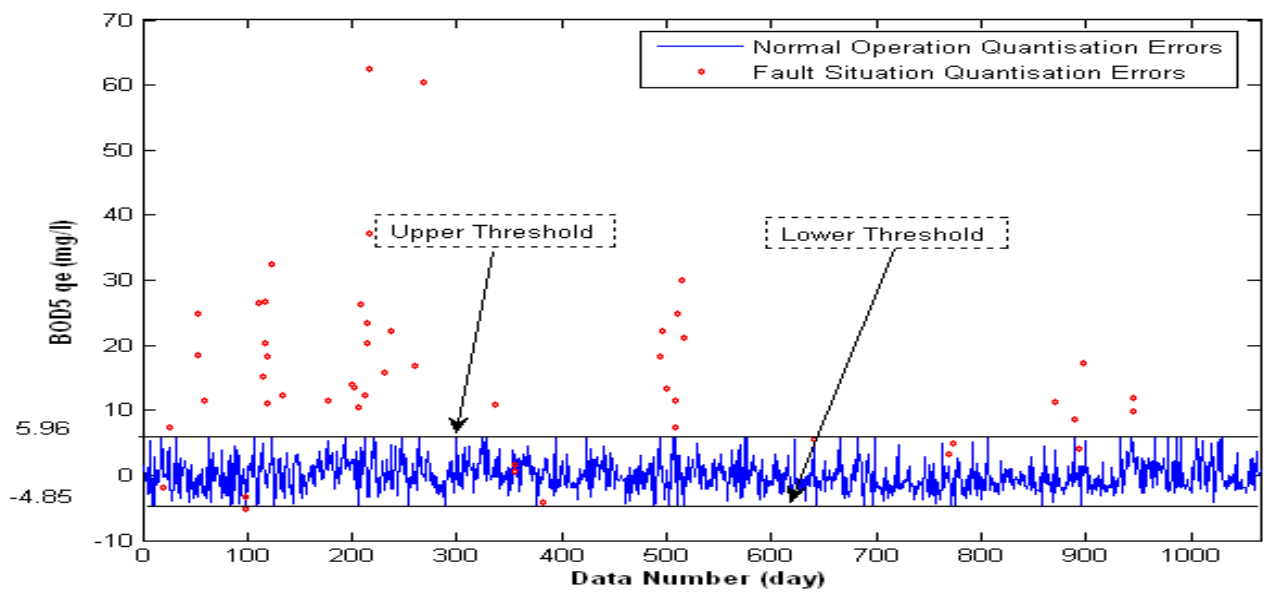

Fig. 1 Quantisation error for BOD5 values
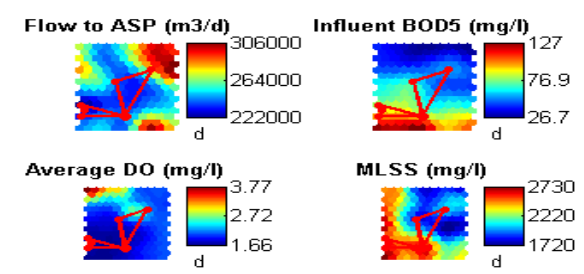

Sludge Age (day)

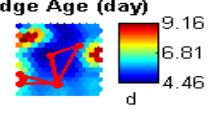

Effluent COD (mg/)

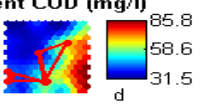

MLSS (mg/l)
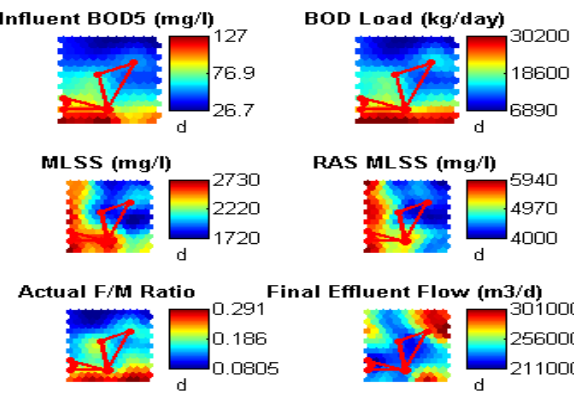

Influent SS to ASP

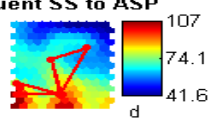

RAS MLSS (mg/l)

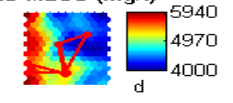

SSVI (mk/g)

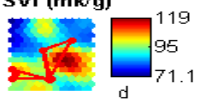

inal Effluent Flow (m3/d)

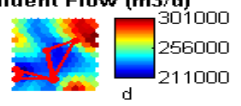

Effluent BOD5 (mg/)

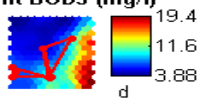

Effluent SS (mg/1)

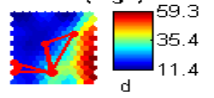

Fig. 2: The Trajectory of the Best Matching Units for Some of the Faulty Data

\section{References}

[1] R. Rustum, "Modelling activated sludge wastewater treatment plants using artificial intelligence techniques (fuzzy logic and neural networks)," 2009.

[2] S. Mani, J. Sundaram, and K. C. Das, "Process simulation and modeling: Anaerobic digestion of complex organic matter," Biomass and Bioenergy, vol. 93, pp. 158-167, 2016.

[3] R. Rustum and A. J. Adeloye, "Replacing outliers and missing values from activated sludge data using kohonen selforganizing map," J. Environ. Eng., vol. 133, no. 9, 2007.

[4] E. P. Tao, W. H. Shen, T. L. Liu, and X. Q. Chen, "Fault diagnosis based on PCA for sensors of laboratorial wastewater treatment process," Chemom. Intell. Lab. Syst., vol. 128, pp. 49-55, 2013.

[5] H. Xiao, D. Huang, Y. Pan, Y. Liu, and K. Song, "Fault diagnosis and prognosis of wastewater processes with incomplete data by the auto-associative neural networks and ARMA model," Chemom. Intell. Lab. Syst., vol. 161, pp. 96-107, 2017.

[6] Z. Baalbaki, E. Torfs, T. Maere, V. Yargeau, and P. A. Vanrolleghem, "Dynamic modelling of solids in a full-scale activated sludge plant preceded by CEPT as a preliminary step for micropollutant removal modelling," Bioprocess Biosyst. Eng., vol. 40, no. 4, pp. 499-510, 2017.

[7] A. Chen, H. Zhou, Y. An, and W. Sun, "PCA and PLS monitoring approaches for fault detection of wastewater treatment process," in IEEE International Symposium on Industrial Electronics, 2016, vol. 2016-Novem, pp. 10221027. 
[8] O. Samuelsson, A. Björk, J. Zambrano, and B. Carlsson, "Gaussian process regression for monitoring and fault detection of wastewater treatment processes," Water Sci. Technol., vol. 75, no. 12, pp. 2952-2963, 2017.

[9] S. Grieu, F. Thiery, A. Traoré, T. P. Nguyen, M. Barreau, and M. Polit, "KSOM and MLP neural networks for on-line estimating the efficiency of an activated sludge process," Chem. Eng. J., vol. 116, no. 1, pp. 1-11, 2006.

[10] R. Rustum, A. J. Adeloye, and M. Scholz, "Applying Kohonen self-organizing map as a software sensor to predict biochemical oxygen demand.," Water Environ. Res., vol. 80, no. 1, pp. 32-40, 2008.

[11] M. Liukkonen, I. Laakso, and Y. Hiltunen, "Advanced monitoring platform for industrial wastewater treatment: Multivariable approach using the self-organizing map,” Environ. Model. Softw., vol. 48, pp. 193-201, 2013.

[12] J. Vesanto, J. Himberg, E. Alhoniemi, and J. Parhankangas, "SOM Toolbox for Matlab 5," Tech. Rep. A57, vol. 2, no. 0, p. 59, 2000. 